\title{
Use of 3D Technology to Support Dermatologists Returning to Practice Amid COVID-19
}

\author{
Stephanie Ishack, PhD; Shari R. Lipner, MD, PhD
}

\section{PRACTICE POINTS}

- Coronavirus disease 19 has overwhelmed our health care system and affected all specialties, including dermatology.

- There are concerns about shortages of personal protective equipment to safely care for patients.

- 3-Dimensional imaging and printing technologies can be harnessed to create face coverings and face shields for the dermatology outpatient setting.

\footnotetext{
${ }^{2}$
} oronavirus disease 2019 (COVID-19) has spread across all 7 continents, including 185 countries, and infected more than 21.9 million individuals worldwide as of August 18, 2020, according to the Johns Hopkins Coronavirus Resource Center. It has strained our health care system and affected all specialties, including dermatology. Dermatologists have taken important safety measures by canceling/deferring elective and nonemergency procedures and diagnosing/treating patients via telemedicine. Many residents and attending dermatologists have volunteered to care for COVID-19 inpatients and donated personal protective equipment (PPE) to hospitals reporting shortages. ${ }^{1}$ As we prepare to treat increasing numbers of in-office patients, there will be a critical need for PPE. We highlight the use of 3-dimensional (3D) imaging and printing technologies as it applies to the dermatology outpatient setting.

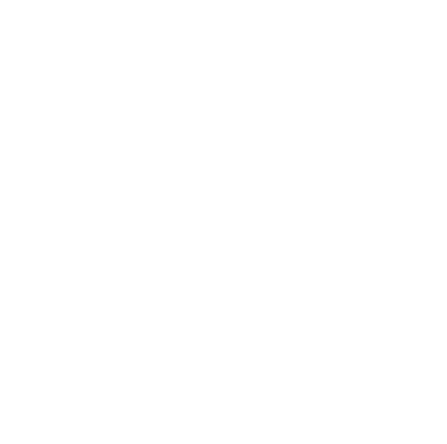

N95 masks are necessary during the COVID-19 pandemic because they effectively filter at least $95 \%$ of $0.3-\mu \mathrm{m}$ airborne particles and provide adequate face seals. ${ }^{1} 3$-Dimensional imaging integrated with 3D printers can be used to scan precise facial parameters (eg, jawline, nose) and account for facial hair density and length to produce comfortable tailored N95 masks and face seals. ${ }^{1,2}$ 3-Dimensional printing utilizes robotics and computeraided design systems to layer and deposit biomaterials, thereby creating cost-effective, customizable, mechanically stable, and biocompatible constructs. ${ }^{1,3}$ An ideal 3D-printed N95 mask would be printed via fused deposition modeling, consisting of a combination of lightweight and fatigue-resistant biomaterials, including electrostatic nonwoven polypropylene and styrene-(ethylenebutylene)-styrene. ${ }^{1,4}$ The resulting masks, made from industrial-grade raw materials, are practical alternatives for dermatology practices with insufficient supplies.

Face shields offer an additional layer of safety for the face and mucosae and also may provide longevity for N95 masks. Using synthetic polymers such as polycarbonate and polyethylene, 3D printers can be used to construct face shields via fused deposition modeling. ${ }^{1}$ These face shields may be worn over N95 masks and then can be sanitized and reused.

Mohs surgeons and staff may be at particularly high risk for COVID-19 infection due to their close proximity to the face during surgery, use of cautery, and prolonged time spent with patients while taking layers and suturing.

Dr. Ishack is from the New York University School of Medicine, New York. Dr. Lipner is from the Department of Dermatology, Weill Cornell Medical College, New York.

The authors report no conflict of interest.

Correspondence: Shari R. Lipner, MD, PhD, 1305 York Ave, New York, NY 10021 (shl9032@med.cornell.edu).

doi:10.12788/cutis.0069 


\section{D Imaging Technology}
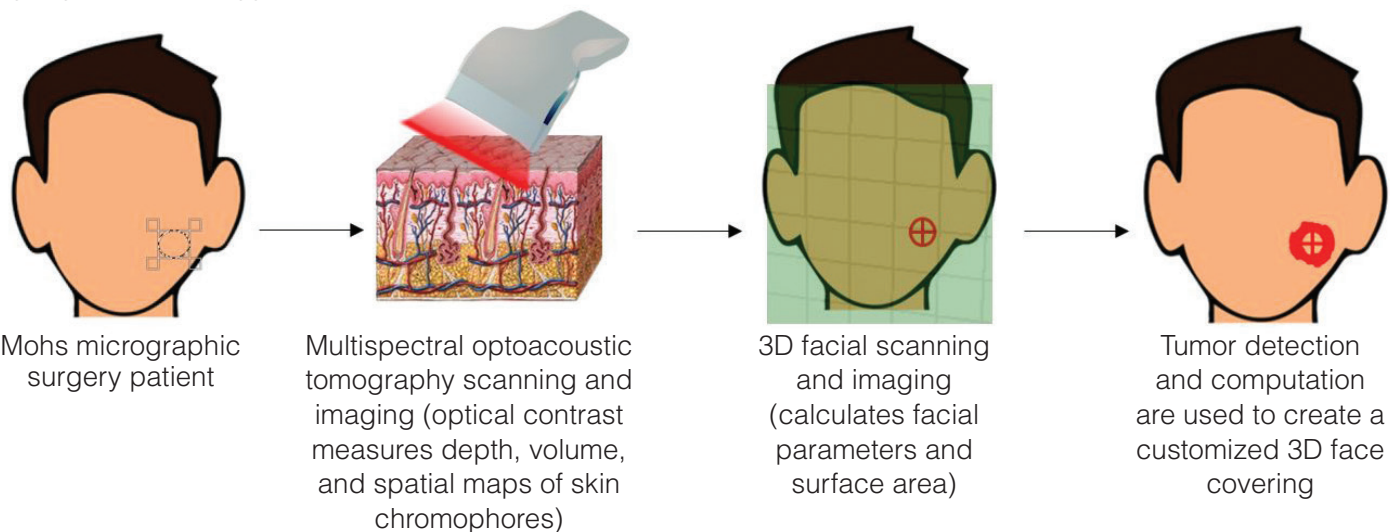

Tumor detection and computation are used to create a customized 3D face covering

\section{D Printing Technology}

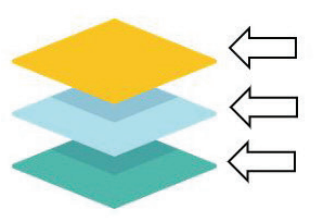

Outer layer of 3D-printed polypropylene

Middle layer of 3D-printed polyester

Inner layer of 3D-printed polypropylene + styrene-(ethylene-butylene)-styrene

\section{D-Printed Mask}

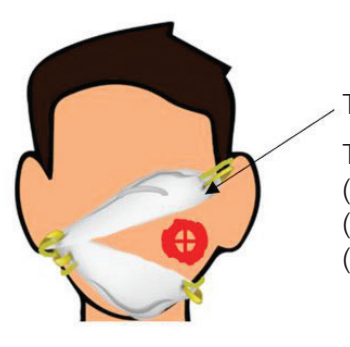

This 3D-printed mask leaves sufficient skin for excision and reconstruction

The customized mask:

(1) Covers the patient's nose and mouth

(2) Only exposes relevant areas near the excision site

(3) Includes adjustable/removable ear loops for tumors localized to the ear

3-Dimensional (3D) imaging technologies and a 3D-printed face covering prototype that can potentially be used on patients during Mohs micrographic surgery. The biomaterials in this diagram have been well tested in the literature and are the same materials that are used in N95 masks.

Multispectral optoacoustic tomography is a noninvasive imaging tool that can map skin tumors via optical contrast with accuracy comparable to histologic measurements. ${ }^{5}$ 3-Dimensional facial imaging and printing can be used to calculate tumor surface area for customized masks, leaving sufficient skin for excision and reconstruction. Patient face coverings would cover the nose and mouth, only expose relevant areas near the excision site, and include adjustable/removable ear loops for tumors localized to the ears. A schematic of how 3D technologies can be applied for Mohs micrographic surgery is provided in the Figure.

As dermatologists reopen and ramp up practice volume, there will be increased PPE requirements. Using 3D technology and imaging to produce N95 masks, face shields, and face coverings, we can offer effective diagnosis and treatment while optimizing safety for dermatologists, staff, and patients.

\section{REFERENCES}

1. Ishack S, Lipner SR. Applications of 3D printing technology to address COVID-19-related supply shortages [published online April 21, 2020]. Am J Med. 2020;133:771-773.

2. Cai M, Li H, Shen S, et al. Customized design and 3D printing of face seal for an N95 filtering facepiece respirator. J Occup Environ Hyg. 2018;3:226-234.

3. Ishack S, Lipner SR. A review of 3-dimensional skin bioprinting techniques: applications, approaches, and trends [published online March 17, 2020]. Dermatol Surg. doi:10.1097/ DSS.0000000000002378.

4. Banerjee SS, Burbine S, Shivaprakash NK, et al. 3D-printable PP/SEBS thermoplastic elastomeric blends: preparation and properties [published online February 17, 2019]. Polymers (Basel). doi:10.3390/polym11020347.

5. Chuah SY, Attia ABE, Long V. Structural and functional 3D mapping of skin tumours with non-invasive multispectral optoacoustic tomography [published online November 2, 2016]. Skin Res Technol. 2017;23:221-226. 